\title{
Cyclin D1 G870A Polymorphism: Relation to the Risk of ALL Development, Prognosis Impact, and Methotrexate Cytotoxicity
}

\author{
Nadia El Menshawy ${ }^{1 *}$, Ahmed B El Marghany ${ }^{1}$, Mohamed M Sarhan², Doaa A \\ Aladle $^{1}$
}

\begin{abstract}
Background: Cyclin D1 (CCND1) regulates cell cycle progression during the late G1 and S phase and takes part in methotrexate metabolism. It was hypothesized that $C C N D 1$ gene polymorphism affects acute lymphoblastic leukemia (ALL) development, prognosis and may relate to methotrexate cytotoxicity. Subjects and methods: This study included 50 ALL patients and 50 healthy controls, CCND1 G870A polymorphism was studied in all items using polymerase chain reaction-restriction fragment length polymorphism (PCR-RFLP) and evaluated methotrexate cytotoxicity for ALL patients using liver function tests before and after methotrexate treatment. We followed up patients for one year to determine disease-free survival (DFS) and overall survival (OS) and its relation to the CCND1 genotype. Results: We found that AA genotype and A allele have a higher risk of developing ALL compared to the control group. Additionally, we found no notable association between CCND1 variant and methotrexate cytotoxicity and no role of CCND1 polymorphism in ALL prognosis. Conclusion: Our results suggested that CCND1 G870A polymorphism is associated with a high risk of ALL development. However, it has no role in ALL prognosis or methotrexate cytotoxicity.
\end{abstract}

Keywords: ALL-Cyclin D1-CCND1- polymorphism- methotrexate

Asian Pac J Cancer Prev, 21 (10), 2941-2947

\section{Introduction}

Childhood acute lymphoblastic leukemia (ALL) represents one of the most common tumors in children with an increased incidence of morbidity and mortality. Genetic susceptibility and exposure to environmental factors play an important role in leukemogenesis (Metayer et al., 2016)

Nowadays, Pharmacogenomics tests, including Genome-wide association studies (GWAS), have a direct identification role in genetic alterations, treatment efficacy, cytotoxicity, prognosis, etc. However, the current knowledge gives little data about how genomic determinants can affect ALL susceptibility, chemotherapy, and chance of relapse (Wu and $\mathrm{Li}, 2018$ ).

Methotrexate (MTX) forms the backbone of maintenance cycles in childhood acute lymphoblastic leukemia (ALL) chemotherapy, including interim maintenance (Mandal et al., 2020). Major adversities include cytopenias, mucositis, liver toxicity, renal toxicity and skin toxicity. These toxicities enquire dose modification or omission (burn out) MTX-related toxicities may be determined by several factors like dose, duration, genetic susceptibility and risk factors (Vaishnavi et al ., 2018).
Cyclin D1 (CCND1) is an essential regulator protein of the eukaryotic cell cycle. CCND1 acts mainly during the G1 phase and forms a complex with CDK4 or CDK6 in the mid to late G1 phase. This complex, activated by CDK-activating kinase, will cause phosphorylation of retinoblastoma protein $(\mathrm{pRb})$. Phosphorylated $\mathrm{Rb}$ liberates transcription factors like E2F, which is responsible for gene transcription required for entry into the $\mathrm{S}$ phase of the cell cycle (Xue et al., 2015). Overexpression of CCND1 unsettles normal cell cycle process and probably stimulate the development and progression of childhood ALL (Kim andDiehl, 2009), colorectal cancer (Xie et al., 2017),cancer risk (Thakur et al., 2018).

A lot of research has been published regarding the effects of genetic polymorphisms on methotrexate (MTX)induced toxicity and efficacy where it is a pivotal drug in the different treatment protocols, both at low and high doses. MTX acts on a variety of target enzymes in the folates cycle, as well as being transported out and into of the cell by several transmembrane proteins (Gervasin and Mota-zamorano., 2019).

Methotrexate (MTX), a folic acid antagonist, is a cornerstone in ALL treatment's maintenance phase. One of MTX mechanisms is that it acts through competitive inhibition of dihydrofolate reductase (DHFR) regulated by 
CCND1 through increased E2F and thus leads to resistance to methotrexate (MTX) or interfere with its cytotoxicity. This leads to reducing the formation of tetrahydrofolate from dihydrofolate, resulting in the lack of the folate coenzymes leading to impairment of DNA and RNA synthesis (Li et al., 2016).

Several gene polymorphism have been investigated concerning susceptibility to ALL including SNPs of carcinogen metabolism genes, folate metabolism genes, DNA repair genes, regulators of lymphoid cell differentiation, tumor suppressors, transcriptional factors and chemokines, in addition, various SNPs participating in the metabolism of antileukemic agents inducing therapy toxicity, discontinuation of therapy with risk of relapse (Kampouraki et al.,2020).

Several studies have revealed that populations with the AA genotype had a significant risk of childhood ALL (Bedewy et al., 2013). Other studies reported the poor outcomes of youth ALL in the 870A allel. Furthermore, studies have shown the relation between methotrexate resistance or toxicity and the studied polymorphism (Xue et al., 2015). To the best of our knowledge, no study has linked the relation between childhood ALL susceptibility, Methotrexate resistance or toxicity, and the chance of relapse in one study.

\section{Materials and Methods}

This study included 50 patients with acute lymphoblastic leukemia aged from one year to 18 years (32 males and 18 females) diagnosed by peripheral blood and bone marrow morphology, immunophenotyping, and cytogenetic analysis. Also, 50 healthy (26 male and 24 female) subjects matched for age and sex served as the control group. All subjects' parents in this study gave written informed consent before being included in the study.

Thirty-two patients with B-ALL received low dose methotrexate, and 18 patients with T-ALL received high dose methotrexate. The study was conducted in the oncology center, Mansoura University, between April 2016 and October 2017. This study was ethically approved by Mansoura faculty of medicine Institutional research board (IRB), Mansoura University.

\section{Genotyping of CCDN1 G870A gene}

Genotyping of the CCDN1 G870A gene was performed using the polymerase chain reactionrestriction fragment length polymorphism (PCR-RFLP) method. First of all, DNA was extracted, it was isolated from EDTA-blood samples by GeneJETTM Whole Blood Genomic DNA Purification Mini Kits from Thermo Scientific (lot no k0781, Lithuania, EU) according to manufacturer guideline. Then, analysis of genetic polymorphism using PCR-RFLP technique by two steps: first amplification of extracted genomic DNA that was done by using PCR via thermal cycler (ARKTIK Thermal Cycler, Thermo Scientific Co.) using a reverse primer (CCND1R-21): TTTCCGTGGCACTAGGTGTC and Forward primer (CCND1F-22) AGTTCATTTCCAATCCGCCC
(BIOSEARCH Tech, Denmark).

PCR was carried as follows: $12.5 \mu \mathrm{l}$ MyTaqTM Red Master Mix (2X) (BioLine Ltd, UK). $1 \mu 1$ DNA template, forward and reverse primers $0.1 \mu \mathrm{l}$ each and $11.3 \mu \mathrm{l}$ nuclease-free water with the final volume of $25 \mu 1$. PCR condition consisted of an initial denaturation of $95^{\circ} \mathrm{C}$ for $1 \mathrm{~min}$, followed by 35 cycles of $94^{\circ} \mathrm{C}$ for $30 \mathrm{sec}, 60^{\circ} \mathrm{C}$ for $30 \mathrm{sec}$, and $72^{\circ} \mathrm{C}$ for $30 \mathrm{sec}$, with a final extension of $72^{\circ} \mathrm{C}$ for $10 \mathrm{~min}$. The PCR product of $212 \mathrm{bp}$ was detected by agarose gel electrophoresis using $2 \%$ agar. Second, the PCR products were then digested with MspI (New England BioLabs Inc, lot no 0551507, UK). The digested PCR products were electrophoresed using 2\% agarose gel to distinguish between $175 \mathrm{bp}$ band produced by the digestion of A allele and the $141 \mathrm{bp}$ produced by the digestion of the $\mathrm{G}$ allele. Heterozygous state yielded both $141 \mathrm{bp}$ and $175 \mathrm{bp}$ bands (Figure 1).

\section{Statistical Methods}

The statistical analysis of the data was performed by using excel (Microsoft office 2013) program and SPSS (Statistical Package For Social Science) program (SPSS, Inc, Chicago, IL) version 20. A Chi-square test was used to compare groups. Quantitative data were presented as median and range, mean and standard deviation. For comparison between two groups, student T-test (for parametric data) and Mann-Whitney test (for non-parametric data) were used. For comparison of more than two groups; One-Way ANOVA (for parametric information) and Kruskal-Wallis (for non-parametric data) were used. For comparison within the same group, paired-sample T-test (for parametric data) and Wilcoxon test (for non-parametric data) were used. The univariate analysis used to evaluate the association of polymorphism with the occurrence of leukemia and hepatotoxicity. Multivariate logistic regression used for hepatotoxicity. For survival analysis, Kaplan-Meier curves were used and compared by the log-rank test.

\section{Results}

The age of the studied patients ranged from 5 to 18 years. Among the 50 patients, 32 (64\%) were males, and $18(36 \%)$ were females. The age of the control group ranged from 3 to 12 years (mean $8.66 \pm 3.50$ ). Among the 50 healthy children, 26 (52\%) were males and 24 (48\%) females. Immunophenotyping of ALL patients revealed 32 (64\%) patients B-ALL and 18 (36\%) patients T-ALL.

In the current study, there was a significant elevation of ALT in T ALL patients compared to control (P-value 0.008). On the other hand, there is no change regarding AST between studied groups. There was also a significant elevation of post-treatment AST (P-value 0.011) and ALT (P-value 0.044) compared to pretreatment in T ALL group. However, there is no significant change in B ALL (Table 1).

Comparison of cyclin D1 variant between cases and control revealed that there was significantly higher AA genotype and A allele in acute leukemia patients when compared to the control group (P-value 0.001), while other genotyping (GG and GA) showed no significant 
Table 1. Comparison of Liver Enzymes in Case Groups before and after Treatment

\begin{tabular}{llccccccc}
\hline & Parameter & \multicolumn{3}{c}{ Pretreatment } & \multicolumn{3}{c}{ Post-treatment } & P \\
& & Median & \multicolumn{2}{c}{ Range } & Median & Range \\
\hline \multirow{3}{*}{ B ALL } & & & Min & Max & & Min & Max \\
& AST & 20 & 4 & 141 & 29 & 15 & 45 & 0.896 \\
& T ALT & 31 & 12 & 98 & 29 & 12 & 45 & 0.071 \\
& AST & 30 & 8 & 129 & 93 & 12 & 1567 & 0.011 \\
& ALT & 39 & 1 & 127 & 85 & 8 & 1258 & 0.044 \\
\hline
\end{tabular}

Wilcoxon test*; *significant (P-value $<0.05)$

Table 2. Comparison of Cyclin D1 Variant between Cases and Control

\begin{tabular}{|c|c|c|c|c|c|c|}
\hline \multirow{2}{*}{$\begin{array}{l}\text { Control }(\mathrm{n}=50) \\
\mathrm{GG}\end{array}$} & & \multirow{2}{*}{$\frac{\text { Acute leukemia }(\mathrm{n}=50)}{13(26 \%)}$} & \multirow{2}{*}{$\frac{P}{0.002}$} & \multirow[t]{2}{*}{ OR } & \multicolumn{2}{|c|}{$95 \% \mathrm{CI}$} \\
\hline & & & & & & \\
\hline $\mathrm{AG}$ & & $25(50 \%)$ & & 1.846 & 0.77 & 4.421 \\
\hline AA & $1(2 \%)$ & $12(24 \%)$ & & 22.154 & 2.584 & 189.958 \\
\hline $\mathrm{AG}+\mathrm{AA}$ & $26(52 \%)$ & $37(74 \%)$ & & 2.627 & 1.133 & 6.092 \\
\hline $\mathrm{AG}+\mathrm{GG}$ & 49 (98\%) & $38(76 \%)$ & & 15.47 & 1.92 & 124.3 \\
\hline AA & $1(2 \%)$ & $12(24 \%)$ & & & & \\
\hline G & $73(73 \%)$ & $51(51 \%)$ & & 2.597 & 1.439 & 4.688 \\
\hline A & $27(27 \%)$ & $49(49 \%)$ & & & & \\
\hline
\end{tabular}

Chi-Square test, odds ratio, CI confidence interval. all data in tables presented as count and percentage; $* *$ significant (P value $<0.05) ; \mathrm{P}$ value of chi square test result from comparing 3 genotypes between two studied groups.

differences between cases and controls. Also, there was a real risk of developing ALL with AA genotype, and the results were highly statistically significant for AA genotype compared to the GG genotype. The study also revealed significant risk for AA genotype compared to non-homozygosity for AA genotype (AG+GG) (Table 2).

Furthermore, the effect of cyclin D1 genotype on the incidence of hepatotoxicity after one week of methotrexate administration as an indicator of methotrexate toxicity were assessed. we didn't find any significant differences between hepatotoxic and non-hepatotoxic patients regarding cyclin D1 genotypes despite a highly significant difference in methotrexate level. (P-value 1.00) (Table 3and4).

Logistic regression analysis was conducted for the prediction of hepatotoxicity in T ALL patients. Age, gender, cyclin D1 genotypes were applied as covariates.
None of the covariates were associated with the risk of hepatotoxicity in univariate analysis. (P-value 1.00), (P-value 0.250) and (P-value 1.00) respectively (Table 5).

No significant differences were found regarding cyclin D1 genotypes in patients with complete remission and partial remission or relapse (Table 6).

At the end of the follow-up period (12 months), the OS of studied ALL cases estimates $98 \%$ at six months and $96 \%$ at 12 months (figure 2 ). As regard disease, free survival was also rated $82 \%$ at five months and $74 \%$ at ten months (figure 3). As regards GG genotype DFS was estimated $78 \%$ at five months and $68 \%$ at ten months. Also, AG genotype was determined $92 \%$ at five months and $80 \%$ at ten months. On the other hand, AA genotype was estimated at $64 \%$ at five months and $64 \%$ at ten months, with no significant difference between 3 genotypes (P-value 0.474) (Figure 4).

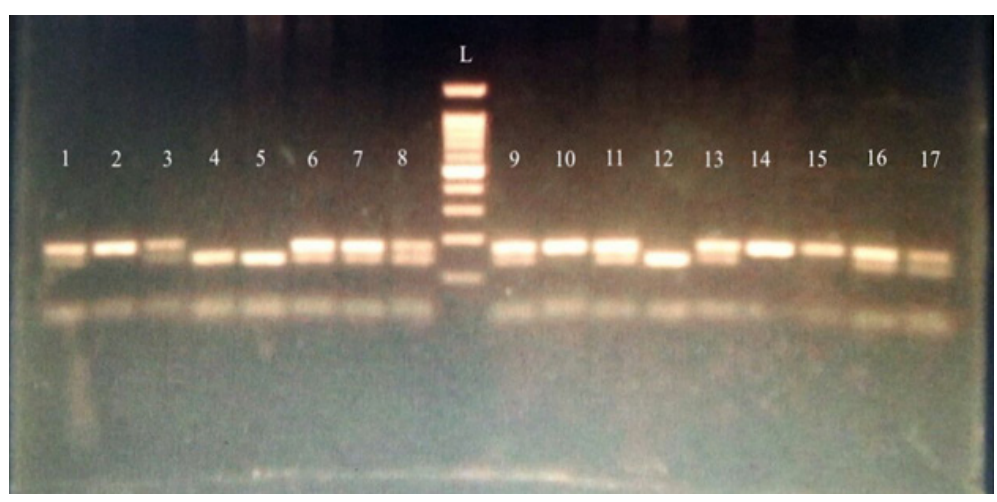

Figure 1. Agarose Gel (2\%) Electrophoresis for RFLP Digested PCR Products. Lanes 2,10,14,15 represents restricted PCR product of an ALL patient with AA genotype (175 bp). Lanes 1,3,6,7,8,9,11,13,16,17 represent restricted fragments of PCR products of ALL patients with AG genotype (175,141 bp). Lanes 4, 5,12 represent restricted PCR products of ALL patients with GG genotype (141bp). Lane (L) represents 100bp ladder base pair marker. 
Table 3. Comparison of Cyclin D1 Variant between Patients with and without Hepatotoxicit

\begin{tabular}{|c|c|c|c|c|c|c|}
\hline \multicolumn{2}{|c|}{ Without hepatotoxicity $(n=7)$} & \multirow{2}{*}{$\frac{\text { Hepatoxicity }(\mathrm{n}=10)}{3(30 \%)}$} & \multirow{2}{*}{$\frac{P}{0.442}$} & \multirow[t]{2}{*}{ OR } & \multicolumn{2}{|c|}{$95 \% \mathrm{CI}$} \\
\hline GG & $1(14.3 \%)$ & & & & & \\
\hline $\mathrm{AG}$ & $5(71.4 \%)$ & $4(40 \%)$ & & 0.267 & 0.019 & 3.653 \\
\hline AA & $1(14 \%)$ & $3(30 \%)$ & & 1 & 0.041 & 24.547 \\
\hline $\mathrm{AG}+\mathrm{AA}$ & $6(85.7 \%)$ & $7(70 \%)$ & & 0.388 & 0.031 & 4.795 \\
\hline $\mathrm{AG}+\mathrm{GG}$ & $6(85 \%)$ & $7(70 \%)$ & & 2.571 & 0.208 & 31.71 \\
\hline AA & $1(14.3 \%)$ & $3(30 \%)$ & & & & \\
\hline G & $7(50 \%)$ & $10(50 \%)$ & & 1 & 0.255 & 3.91 \\
\hline A & $7(50 \%)$ & $10(50 \%)$ & & & & \\
\hline
\end{tabular}

Odds ratio , CI confidence interval. all data in tables presented as count and percentage; ** significant (P value $<0.05)$; $\mathrm{P}$ value of chi square test result from comparing 3 genotypes between two variants.

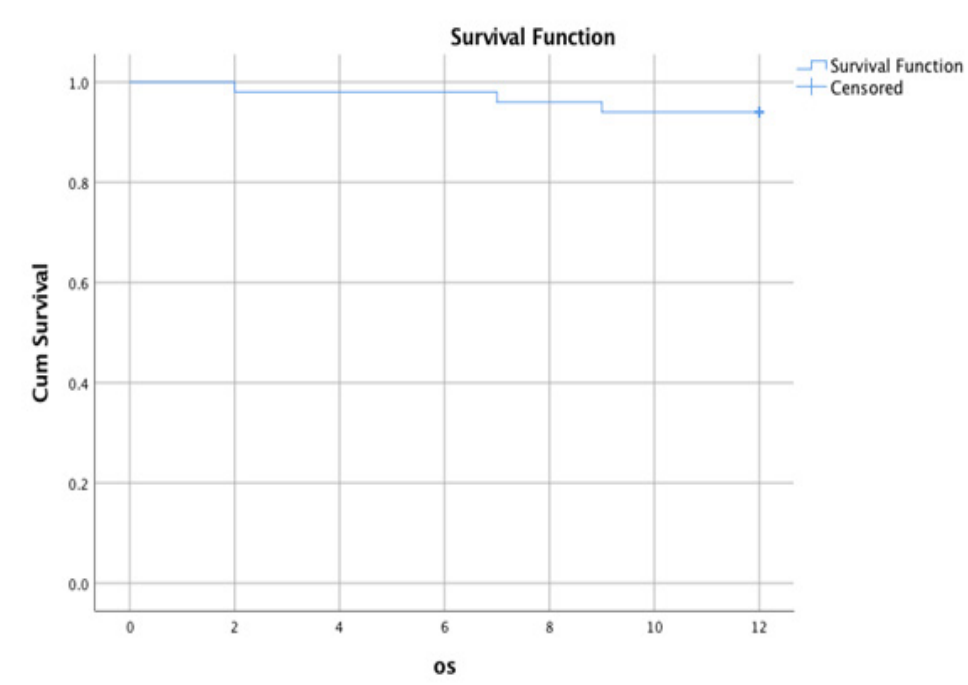

Figure 2. Overall Survival (OS) of Studied ALL Cases

\section{Discussion}

Acute lymphoblastic leukemia (ALL) is the most common cancer in children, and although the cure rates exceed $90 \%$, it remains a significant cause of morbidity and mortality in children and adults. The past decade has been marked by extraordinary advances in the genetic basis of leukemogenesis and treatment responsiveness in ALL (Iacobucci andMullighan, 2017).

Till now, over 50 years have gone after MTX was first presented in the clinic. It has been involved in most of the treatment protocols of many solid tumors, including hematological malignancies, including ALL. High-dose methotrexate (HDMTX) refers to infused MTX in doses of more than $1 \mathrm{~g} / \mathrm{m}^{2}$. Using HDMTX shows the marvelous result in treating ALL and prevention of extramedullary leukemia (i.e., central nervous system leukemia and testicular leukemia), as MTX can penetrate the blood- brain barrier and blood-testis barrier (Qui et al.,2017.

The primary target of MTX is the enzyme dihydrofolate reductase (DHFR), which can catalyze the reduction of folate and 7, 8-dihydrofolate to 5, 6, 7, 8-tetrahydrofolate. This will lead to the impairment of nucleic acid synthesis and cellular death. Many proteins involved in the folate metabolic pathway (i.e., MTHFR, RFC, TS) may contribute to MTX cytotoxicity and clinical elimination (Liu et al.,2017).

Cyclin D1 is involved in the folate metabolic pathway and has been shown to influence cellular response to MTX. Thus CCND1 influences the clearance of MTX and MTX-associated toxicity partly through the regulation of DHFR and THF (Xue et al., 2015).

The present study revealed a highly significant association between A allele either in heterozygous (AG) or homozygous state (AA) and cases. Besides, there was a real risk of developing ALL with AA genotype, and

Table 4. Comparison of Methotrexate Level at 48 Hours after Administration between Patients with and without Hepatotoxicity

\begin{tabular}{|c|c|c|c|c|c|c|c|}
\hline \multirow[t]{3}{*}{ Parameter } & \multicolumn{3}{|c|}{ Without hepatotoxicity $(\mathrm{n}=7)$} & \multicolumn{3}{|c|}{ Hepatoxicity $(\mathrm{n}=10)$} & \multirow[t]{3}{*}{$\mathrm{P}$} \\
\hline & \multirow[t]{2}{*}{ Median } & \multicolumn{2}{|c|}{ Range } & \multirow[t]{2}{*}{ Median } & \multicolumn{2}{|c|}{ Range } & \\
\hline & & Min & Max & & Min & Max & \\
\hline Methotrexate (umol/L) & 0.5 & 0.2 & 0.8 & 2.75 & 1.9 & 4 & 0.001 \\
\hline
\end{tabular}


Table 5. Logistic Regression Analysis for Prediction of Hepatotoxicity within T ALL Patients

\begin{tabular}{|c|c|c|c|c|c|c|c|}
\hline \multicolumn{2}{|l|}{ Parameter } & \multirow{3}{*}{$\begin{array}{c}\text { No hepatotoxicity } \\
1\end{array}$} & \multirow{3}{*}{$\begin{array}{c}\text { Hepatotoxicity } \\
2\end{array}$} & \multicolumn{3}{|c|}{ Univariate } & \multirow{3}{*}{$\frac{P}{1}$} \\
\hline & & & & \multirow{2}{*}{$\frac{\mathrm{OR}}{0.667}$} & \multicolumn{2}{|c|}{$95 \% \mathrm{CI}$} & \\
\hline Age & $<5$ years & & & & 0.048 & 9.189 & \\
\hline & $>5$ years & 6 & 8 & & & & \\
\hline \multirow[t]{2}{*}{ Gender } & Male & 4 & 9 & 0.148 & 0.012 & 1.9 & 0.25 \\
\hline & Female & 3 & 1 & & & & \\
\hline \multirow[t]{3}{*}{ Cyclin D1 genotype } & GG & 1 & 3 & & & & \\
\hline & AG & 5 & 4 & 0.267 & 0.019 & 3.653 & 0.308 \\
\hline & AA & 1 & 3 & 1 & 0.041 & 24.547 & 1 \\
\hline
\end{tabular}

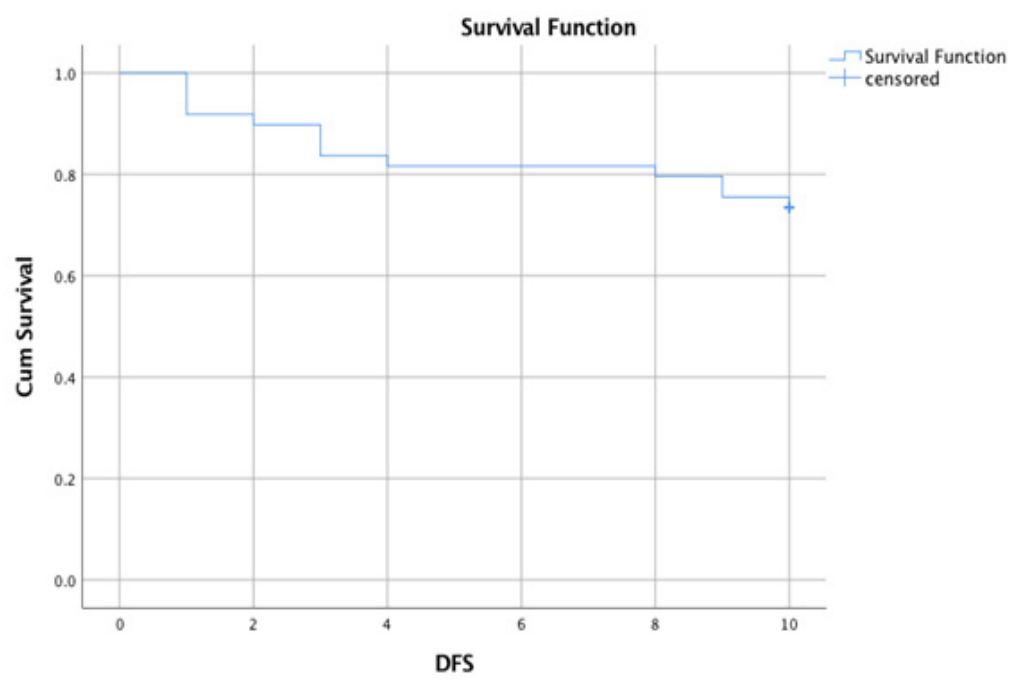

Figure 3. DFS of Studied ALL Cases

the results were highly statistically significant for AA genotype compared to the GG genotype. These findings were concordant with those of Hou et al., (2005), who studied 183 ALL cases and 190 age and sex-matched healthy controls, and found that CCND1 A allele was more frequent in the ALL group than in the control group. Hou et al., (2005) and Bedewy et al., (2013) who studied the association of CCND1 $6870 \mathrm{~A}$ polymorphism with ALL risk in 25 ALL patients and 15 healthy controls and found that frequency of the AA genotype was significantly increased in the ALL cases while GG genotype was significantly increased in the control group. In more addition significant association between CCND1 G870A polymorphism and Nasopharangyl carcinoma risk was found in the central-southern Chinese population (Liao et al., 2014).

The study also revealed significant risk for AA genotype when compared to non-homozygosity for AA genotype ( $\mathrm{AG}+\mathrm{GG})$ in contrast to Hou et al., (2005) and Bedewy et al., (2013). The impact of the CCND1 A allele on childhood ALL was thought to be due to a higher level of CCND1b resulting in a longer half-life protein, which might affect the transition from the G1 to S phase of cell cycle and in turn cell proliferation (Knudsen, 2006)

Because ALL patients were administrated with MTX in different doses $\left(5.0 \mathrm{~g} / \mathrm{m}^{2}\right.$ for children with high-risk or medium-risk ALL and $3.0 \mathrm{~g} / \mathrm{m}^{2}$ for children with low-risk ALL), a stratification analysis was conducted.

Relations between clinical response and systematic toxicity after MTX treatment and gene variation in the

Table 6. Comparison of Cyclin D1 Variant between Partial Remission or Relapse and Complete Remission

\begin{tabular}{|c|c|c|c|c|c|c|c|}
\hline & & Complete remission $(n=35)$ & Partial remission or relapse $(n=14)$ & $\mathrm{P}$ & OR & \multicolumn{2}{|c|}{$95 \% \mathrm{CI}$} \\
\hline GG & Count, $\%$ & $9(25.7 \%)$ & $4(28.6)$ & 0.987 & & & \\
\hline $\mathrm{AG}$ & Count, $\%$ & $18(51.4 \%)$ & $7(50 \%)$ & & 0.875 & 0.202 & 3.791 \\
\hline AA & Count, $\%$ & $8(22.9 \%)$ & $3(21.4)$ & & 0.844 & 0.143 & 4.974 \\
\hline $\mathrm{AG}+\mathrm{AA}$ & Count, $\%$ & $26(74.3 \%)$ & $10(71.4 \%)$ & & 0.865 & 0.216 & 3.458 \\
\hline $\mathrm{AG}+\mathrm{GG}$ & Count, \% & $27(77.1 \%)$ & $11(78.5 \%)$ & & 0.920 & 0.205 & 4.128 \\
\hline AA & Count, $\%$ & $8(22.9 \%)$ & $3(21.5 \%)$ & & & & \\
\hline G & Count, $\%$ & $36(51.4 \%)$ & $15(53.6 \%)$ & & 0.917 & 0.381 & 2.208 \\
\hline A & Count, $\%$ & $34(48.6 \%)$ & $13(46.4 \%)$ & & & & \\
\hline
\end{tabular}

$\mathrm{P}$ value of chi square test result from comparing 3 genotypes between two; ${ }^{* *}$ significant $(\mathrm{P}$ value $<0.05)$ 


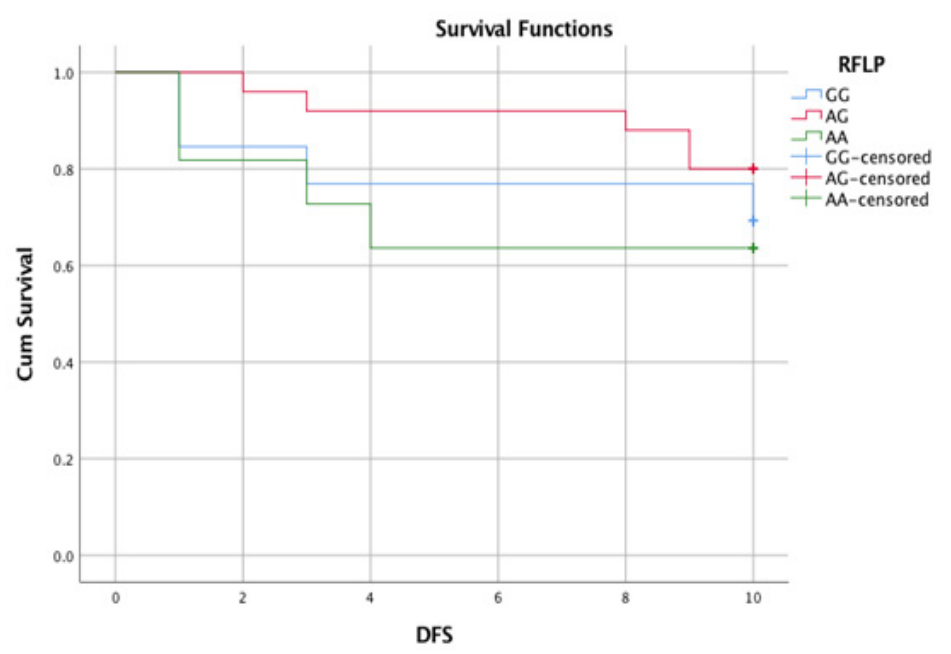

Figure 4. DFS in Relation to cyclin D1 Variant

folate metabolic pathway have been detected many times Most studies have focused on MTHFR C677T and RFC G80A and suggested that these polymorphisms contribute to MTX therapy-related toxicity or outcome of childhood ALL (Umerez et al., 2017, Tiwari et al., 2018).

In this study, we studied the effect of cyclin D1 genotype on the incidence of hepatotoxicity after one week of methotrexate administration as an indicator of methotrexate toxicity. We didn't find any significant differences between hepatotoxic and non-hepatotoxic patients regarding cyclin D1 genotypes.

The results of the current study were matched with results of Dulucq et al., (2008) who did not find any association between toxicity parameters investigated and the increasing number of event-predisposing genotypes DHFR haplotype*1, CCND1 870AA, and TS 3R3R. But they thought this was most likely because in these three at-risk genotypes, only the CCND1, but not TS and DHFR, were individually associated with a lower frequency of toxic events (Dulucq et al., 2008).

However, in contrast with the results of the present study, Costea et al. (2006) observed that individuals with CCND1 870AA genotype had lower liver toxicity rates. They also found significantly lower rates of these toxicities in individuals with a combination of the CCND1 870AA and MTHFR 677 TT/CT genotypes. This observation may be explained by the variant isoform of CCND1 with a longer half-life and the correlated higher level of DHFR (Costea et al., 2006).

Furthermore, Xue et al., (2015) have found that subjects with $870 \mathrm{AG}$ and $870 \mathrm{AA}$ genotypes have significantly higher rates of MTX-related hepatotoxicity. They also found a statistically significant relationship between the hepatotoxicity and the combined genotypes AG/AA, suggesting that the $870 \mathrm{~A}$ allele may be a risk factor of toxic events. Their subgroup analysis showed that the association of $G 870 \mathrm{~A}$ polymorphism and an increased risk of MTX-related hepatotoxicity was more pronounced in children administrated with MTX in a dose of $5.0 \mathrm{~g} / \mathrm{m} 2$ (high-risk ALL and medium risk ALL). This discrepancy may be due to different genetic backgrounds and population-specific differences (Xue et al., 2015).
After following up ALL patients for one year, there was no statically significant difference among CCND1 genotypes regarding overall survival and event-free survival (P-value 0.474$)$. However, there was a clinically significant association between CCND1 AA genotype and poor survival outcome compared to $A G$ and $G G$ genotypes in contrast to Costea et al., (2006) who studied 205 children of French-Canadian origin with ALL and follow them up for 60 months, the analysis of the impact of CCND1 showed that the individuals with AA variant had a remarkably lower probability of 5-year post-treatment EFS compared to those with AG and GG genotypes (37\% compared to $88 \%, \mathrm{P}<0.00005$ ).

Moreover, some other confounding factors may interfere with the present results, such as some unknown gene variations in the folate metabolic pathway. At the same time, the fact cannot be ignored that our chemotherapy regimen is not identical to other research institutions ( Dulucq et al., 2008; Kantar, 2009) and some chemotherapeutic drugs used in our center may also influence the evaluation of MTX-related hepatotoxicity.

We are studying genetic factors aid in early diagnosis and treatment of ALL. CCND1 AA genotype and A allele are associated with high risk to develop ALL. Cyclin D1 polymorphism may not be contributed to MTX induced hepatotoxicity and couldn't be useful in the clinical prediction of prognosis.

The sample size was relatively small, so it led to a larger span of $95 \%$ CI (0.041 - 24.547). Further studies with a large sample size are needed to confirm the relationship between gene polymorphism and MTX induced hepatotoxicity. In the present study, one laboratory index was only explored (serum aminotransferase). In further studies, other toxicities such as oral mucositis and vomiting should be observed, and the Serum level of MTX should be involved to assess MTX clearance association with CCND1 genotype.

\section{Acknowledgments}

A lot of thanks and greeting for prof Doaa Al Adel for her kind and meticulous revision and support for 
continuous work. A lot of thanks for laboratory assistant for her effort.

\section{References}

Bedewy AM, Mostafa MH, Saad AA, et al (2013). Association of Cyclin D1 A870G polymorphism with two malignancies. Acute lymphoblastic leukemia and breast cancer. $J B U O N$, 18, 227-38

Costea I, Moghrabi A, Laverdiere C, Graziani A, Krajinovic M (2006). Folate cycle gene variants and chemotherapy toxicity in pediatric patients with acute lymphoblastic leukemia. Haematologica, 91, 1113-6.

Dulucq S, St-Onge G, Gagné V, et al (2008). DNA variants in the dihydrofolate reductase gene and outcome in childhood ALL. Blood, 111, 3692-0.

Gervasini G, Mota-Zamorano S (2019). Clinical implications of methotrexate pharmacogenetics in childhood acute lymphoblastic leukaemia. Curr Drug Metabolism, 20, 4.

Hou X, Wang S, Zhou Y, et al (2005). Cyclin D1 gene polymorphism and susceptibility to childhood acute lymphoblastic leukemia in a Chinese population. Int $J$ Hematol, 82, 206-9.

Iacobucci I, Mullighan CG (2017). Genetic basis of acute lymphoblastic leukemia. J Clin Oncol, 35, 975-83.

Kampouraki E,Goulielmos NG, Stiakaki E(2020).Understanding the role of genetics in childhood acute lymphoblastic leukemia (Review). World Academy Sci J, 13, 313-30.

Kantar M, Kosova B, Cetingul N, et al (2009). Methylenetetrahydrofolate reductase C677T and A1298C gene polymorphisms and therapy-related toxicity in children treated for acute lymphoblastic leukemia and non-Hodgkin lymphoma. (2009). Leuk Lymphoma, 50, 912-7.

Kim JK, Diehl JA (2009). Nuclear cyclin D1: An oncogenic driver in human cancer. J Cell Physiol, 220, 292-6.

Knudsen KE (2006). The cyclin D1b splice variant: an old oncogene learns new tricks. Cell Division, 1, 1-12.

$\mathrm{Li}, \mathrm{X}$ (2016). The association between reduced folate carrier-1 gene $80 \mathrm{G} / \mathrm{A}$ polymorphism and methotrexate efficacy or methotrexate related-toxicity in rheumatoid arthritis: A meta-analysis. Int Immunopharmacol, 38, 8-15.

Liao D, Wu Y, Pu X, et al (2014). Cyclin D1 G870A polymorphism and risk of nasopharyngeal carcinoma: A case-control study and meta-analysis. PLoS One, 2014, 9 , e113299.

Liu SG, Gao C, Zhang RD, et al (2017). Polymorphisms in metho trexate transporters and their relationship to plasma methotrexate levels, toxicity of high dose methotrexate, and outcome of pedi atric acute lymphoblastic leukemia. Oncotarget, 2017, 37761-72.

Mandal P, Samaddar S, Chandra J, Parakh N, Goel M (2020). Adverse effects with intravenous methotrexate in children with acute lymphoblastic leukemia/lymphoma: a retrospective study. Indian J Hematol Blood Transfus, 36, 498-504

Metayer C, Dahl G, Wiemels J, Miller M (2016). Childhood leukemia: A preventable disease. Pediatrics, 138, 45-55.

Qui Q,Huang J,Shu X, et al (2017). Polymorphisms and Pharmacogenomics for the Clinical Efficacy of Methotrexate in Patients with Rheumatoid Arthritis: A Systematic Review and Meta-analysis. Sci Rep, 7, 44015.

Thakur N, Kumari S, Mehrotra R (2018). Association between Cyclin D1 G870A (rs9344) polymorphism and cancer risk in Indian population: meta-analysis and trial sequential analysis. Biosci Rep, 38, BSR20180694.

Tiwari P, Ganesan P, Radhakrishnan V, et al (2018). Prospective evaluation of the toxicity profile, and predictors of toxicity of high dose methotrexate in patients of acute lymphoblastic leukemia/lymphoma. Pediatr Hematol Oncol J, 3, 1-5.

Umerez M, Gutierrez Camino Á, Muñoz Maldonado C, Martin Guerrero I, Garcia Orad A (2017). MTHFR polymor phisms in childhood acute lymphoblastic leukemia: Influence on methotrexate therapy. Pharmgenomics Pers Med, 10, 69- 78.

Vaishnavi K, Bansal D, Trehan A, Jain R, Attri SV (2018). Improving the safety of high-dose methotrexate for children with hematologic cancers in settings without access to MTX levels using extended hydration and additional leucovorin. Pediatr Blood Cancer, 16, e27241.

Wu C, Li W (2018). Genomics and pharmacogenomics of pediatric acute lymphoblastic leukemia. Crit Rev Oncol Hematol, 126, 100-11.

Xie M, Zhao F, Zou X, Jin S, Xiong S (2017). The association between CCND1 G870A polymorphism and colorectal cancer risk: A meta-analysis. Medicine (Baltimore), 96, e8269.

Xue Y, Rong L, Tong N, et al (2015). CCND1 G870A polymorphism is associated with toxicity of methotrexate in childhood acute lymphoblastic leukemia. Int J Clin Exp Pathol, 8, 11594.

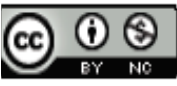

This work is licensed under a Creative Commons AttributionNon Commercial 4.0 International License. 